\title{
HORMONAS Y MIGRAÑA, UNA APROXIMACIÓN CLÍNICA
}

\author{
Leonardo Hernández.MD*, Karen Marín MD**
}

\section{Resumen}

La migraña es la cefalea primaria más frecuente en las mujeres y si se suman las diversas fluctuaciones hormonales en el transcurso de la vida, la prevalencia es hasta tres veces más que en los hombres. Varía según la edad sexual siendo muchas veces la menarquia el inicio de la misma, así como la presentación exclusiva o facilitada por el período menstrual, la posmenopausia o el verdadero reto, el embarazo. Cuando mujeres de diferentes edades y tipos de cefalea, consultan, es la experticia y la adecuada orientación clínica inicial las que pueden lograr un diagnóstico oportuno y acertado. La migraña es un dolor de cabeza en los servicios de urgencias, no solo por la complejidad de su manejo en las pacientes gestantes, sino por las pocas opciones terapéuticas disponibles que sean seguras más para el feto que para la madre. El médico se ve obligado a tomar decisiones terapéuticas acertadas, generando mejoría del cuadro clínico y confianza en su paciente. Abordamos entonces el dolor de cabeza migrañoso en las mujeres durante las diversas edades en que la fluctuación de hormonas sexuales genera este trastorno, desde los puntos de vista fisiopatológico, enfoque diagnóstico y principales opciones terapéuticas estudiadas hasta el momento.

Palabras clave: migraña, menstruación, embarazo, hormonas, tratamiento.

Abreviaturas: MG, migraña (s); ACV, accidente cerebrovascular.

\section{HORMONES AND MIGRAINE - A CLINICAL APPROACH}

\section{Abstract}

\begin{abstract}
Migraine is the most common primary headache in women and fluctuating hormone levels during their lifetime is a contributing factor. It is three times more common in females than males. It varies according to sexual age and many times it begins with menarche, as well as may be affected exclusively or facilitated by menstrual cycles, menopause or pregnancy, constituting the actual challenge. When women of various ages and types of headache consult, expertise ensures a timely and accurate diagnosis. Migraine is a nuisance in the emergency services, not only due to the complexity of its management in pregnant women, but for the few available therapeutic options which are safer for the unborn baby than for the mother. The physician must determine appropriate therapies, achieving improvement and gaining patient's confidence. We approach migraine in women during different ages in which sexual hormone fluctuation is a contributing factor from a pathophysiologic and diagnostic point of view, and current major therapeutic options evaluated to date.
\end{abstract}

Key words: migraine, menstruation, pregnancy, hormones, treatment

Fecha recibido: febrero 2 de 2015 - Fecha aceptado: febrero 24 de 2015

* Coordinador Académico de Neurología. Hospital de San José. Instructor Asistente, Fundación Universitaria de Ciencias de la Salud. Bogotá DC, Colombia.
** Residente III de Neurología. Fundación Universitaria de Ciencias de la Salud. Bogotá DC, Colombia. 


\section{Fisiopatología}

La migraña es tres veces más frecuente en las mujeres ${ }^{1}$ y la mayoría se quejan de intensificación de sus ataques durante el período menstrual o perimenstrual. Es entonces cuando en forma precisa debe clasificarse este padecimiento con el ánimo de entender si es exclusivo de presentación menstrual o precipitada por el mismo.

Durante el ciclo menstrual hay una fluctuación hormonal fisiológica que parece desencadenar un estímulo suficiente para generar un ataque de $\mathrm{MG}^{2}$, el cual tiene más probabilidades de ocurrir durante las fases folicular y lútea temprana, cuando los niveles de estrógeno caen o son bajos ${ }^{3,4}$, por lo que el mantenimiento de los mismos evita su aparición como sucede en las mujeres que toman anticonceptivos orales combinados, quienes experimentan el ataque a la semana de terminar los 21 días de píldoras, o durante el embarazo cuando los niveles de estrógeno aumentan en forma gradual y hay mejoría del dolor, o en la posmenopausia cuando los niveles permanecen bajos y no hay fluctuación hormonal..$^{5-8}$

Por otro lado, el estrógeno altera la expresión de genes que codifican para las proteínas que median el dolor ${ }^{9}$, aumentando la producción de péptido relacionado con el gen de la calcitonina (CGRP) que es bien conocido por estar implicado en la modulación del dolor en el núcleo caudal del trigémino, así como un aumento en la producción de óxido nítrico a través de la activación de los receptores de estrógeno (ER-a), que ha demostrado promover la producción de mediadores proinflamatorios y activar los aferentes trigeminales. ${ }^{10-12}$

Se ha sugerido que la mejoría podría también estar relacionada con cambios en el metabolismo de la serotonina y un aumento de las concentraciones de endorfinas durante los últimos dos trimestres del embarazo. ${ }^{13}$ Además las pacientes con MG tienen niveles de serotonina más bajos entre ataques ${ }^{14}$ y se conoce que su síntesis está influenciada por las hormonas ováricas. ${ }^{15} \mathrm{El}$ estradiol y la progesterona podrían tener efectos opuestos sobre los neurotransmisores, el primero aumenta la actividad del receptor de glutamato NMDA, mientras que la progesterona aumenta la conductancia de cloruro mediada por el GABA, lo que parece tener un papel en la susceptibilidad a la MG. ${ }^{16}$

La activación de los mastocitos durales durante el ciclo se ha demostrado implicada en la génesis de la MG y este efecto fue abolido con la esplenectomía, lo que sugiere una relación de los estrógenos con las células cebadas del bazo, lo que podría explicar de alguna manera la mayor frecuencia de MG en las mujeres, así como las fluctuaciones en el dolor de cabeza durante el ciclo menstrual. ${ }^{17,18}$

Otros mecanismos que podrían estar envueltos se relacionan con la dopamina y los opioides para la MG menstrual, incluyendo la reducción de las concentraciones de beta-hidroxilasa dopamina en el suero ${ }^{19}$, la disfunción del control de opioides del eje hipotalámico-pituitario-adrenal ${ }^{20}$ y la reducción de los niveles de magnesio. ${ }^{21}$

\section{Diagnóstico}

El ámbito internacional acepta la clasificación de la IHSD (International Headache Society Definitions) cuya última actualización se realizó en 2013 y establece los criterios diagnósticos en el mundo de la neurología del dolor de cabeza, ya que se cumplen en cerca de $60 \%$ de los casos. ${ }^{22}$ Hay que conocer, entender e interpretar en forma adecuada los criterios de $\mathrm{MG}$ con y sin aura antes de pasar a los relacionados con las fluctuaciones hormonales, pues estos son la base de la pirámide diagnóstica.

\section{Migraña sin aura}

A. Al menos cinco ataques que cumplan los criterios B y D.

B. Duración de los ataques de cefalea de 4 a 72 horas (no tratada o tratada sin éxito).

C. Cefalea que tenga por lo menos dos de las siguientes características: 1) localización unilateral; 2) pulsátil; 3) dolor de moderada a severa intensidad 
y 4) empeora con la actividad física (caminar o subir escaleras).

D. Durante la cefalea al menos una de las siguientes características: 1) náusea y/o vómito y 2) fono y fotofobia.

E. No se explica mejor por otro diagnóstico de la IHSD-3.

\section{Migraña con aura}

A. Al menos dos ataques que cumplan los criterios B y C.

B. Uno o más de los siguientes síntomas de aura que sean por completo reversibles:1) visual; 2) sensorial; 3) discurso o lenguaje; 4) motor; 5) tallo cerebral y 6) retina

C. Al menos dos de las siguientes cuatro características: 1) al menos un síntoma de aura que se extienda en forma gradual por más de cinco minutos y/o dos o más síntomas que pueden ocurrir de manera sucesiva; 2) cada síntoma individual de aura dura entre 5 y 60 minutos; 3) al menos uno de los síntomas de aura es unilateral y 4) el aura está acompañada o seguida dentro de los siguientes 60 minutos por cefalea.

D. No se explica mejor por otro diagnóstico ICHD-3 y el ataque isquémico transitorio ha sido excluido.

E. Una vez estudiados y comprendidos estos criterios, se proponen los relacionados con los cambios hormonales de la siguiente manera.

\section{Migraña menstrual pura sin aura}

A. Ataque durante la menstruación que cumpla con los criterios de MG sin aura y el siguiente criterio B.

B. Registro con evidencia documentada y prospectiva de al menos tres ciclos consecutivos que confirmen como los ataques se producen solo en el día -2 a +3 de menstruación en al menos dos de tres ciclos menstruales y en ningún otro momento del ciclo.

Se estima que tienen verdadera MG menstrual el 7\% de las mujeres con MG y $11 \%$ de aquellas que la de- sarrollan al inicio de la menarquia ${ }^{23}$, que son las más propensas a relacionarlas con la menstruación. ${ }^{24}$

\section{Migraña con aura relacionada con la menstruación}

A. Ataques en una mujer que está menstruando que cumpla los criterios de migraña sin aura y el siguiente criterio B.

B. Registro de evidencia documentada y prospectiva de al menos tres ciclos consecutivos que confirmen como los ataques se producen solo el día -2 a +3 de la menstruación en al menos dos de tres ciclos menstruales y además en otros momentos del ciclo.

La MG asociada con la menstruación puede ocurrir sola o como parte del síndrome premenstrual (trastorno disfórico de la fase lútea tardía). Aquellas con síndrome premenstrual son más susceptibles a los dolores de cabeza severos no migrañosos tanto durante la fase premenstrual como en todo el ciclo menstrual. ${ }^{25} \mathrm{La}$ MG que aparece por primera vez durante el embarazo (una ocurrencia poco frecuente) o es inducida por la ingesta de anticonceptivos orales (altos niveles de estrógeno) es a menudo la que cursa con aura.

\section{Migraña no menstrual sin aura}

A. Ataques durante la menstruación que cumpla los criterios de migraña sin aura y el B siguiente.

B. Los ataques no cumplen con los criterios para MG menstrual pura sin aura o sin aura relacionada con la menstruación.

La importancia de distinguir entre MG menstrual pura sin aura y la sin aura relacionada con la menstruación radica en la eficacia de la profilaxis hormonal en esta última. La MG menstrual ocurre en o entre los días -2 a +3 del ciclo menstrual y en ningún otro momento..$^{26,27}$

\section{Epidemiología}

El embarazo puede cambiar el patrón de una MG establecida y entre $50 \%$ y $90 \%$ puede mejorar e in- 
cluso desaparecer durante el mismo, sobre todo en el segundo y tercer trimestres ${ }^{28-30}$, y en especial cuando se relaciona con la menstruacion ${ }^{31}$, pero $20 \%$ de las pacientes con MG no tienen ningún cambio en el patrón de dolor de cabeza y $10 \%$ tienen su primer ataque durante el embarazo. ${ }^{28,32}$

Sólo de $4 \%$ a $8 \%$ de las migrañosas empeoran durante el embarazo, sobre todo en las que tienen MG con aura. La lactancia materna parece ejercer un efecto profiláctico en el período posparto ${ }^{33}$, mientras que la alimentación con biberón se asocia con un retorno acelerado de los síntomas. ${ }^{29}$ Es posible que la MG pueda aumentar en frecuencia e intensidad en los períodos pre y perimenopáusico ${ }^{34}$ debido a la secreción errática de estrógenos en esos momentos. ${ }^{35}$ Después de la menopausia, cuando los niveles de hormonas sexuales se estabilizan, la mayoría experimentan una mejoría. ${ }^{36}$

El uso de la terapia de reemplazo hormonal por vía oral puede exacerbar la $\mathrm{MG}^{37}$, tal vez debido a los niveles de absorción y del plasma impredecibles. ${ }^{38}$ Sin embargo, en un análisis multivariado de 18.221 posmenopáusicas tratadas con terapia hormonal, la dosis intermedia de estrógeno de $0.625 \mathrm{mg} /$ día se asoció con una reducción de la frecuencia de cefalea en comparación con dosis más altas o más bajas. ${ }^{39}$ En contraste con la terapia oral, la transdérmica de sustitución hormonal no parece exacerbar la MG y puede incluso tener efectos preventivos ${ }^{40,41}$, de nuevo es posible por la absorción más estable y menos fluctuación en los niveles plasmáticos hormonales.

\section{Pronóstico y complicaciones}

$\mathrm{El}$ riesgo de $\mathrm{ACV}$ es seis veces mayor para la $\mathrm{MG}$ con aura $^{42}$ que la sin aura. En un estudio, las MG se asociaron con un mayor riesgo de ACV isquémico (OR, 3,54 para todas las MG 3,81 para aquellas con aura y 2,97 sin aura), pero no de ACV hemorrágico (OR, 1,1). ${ }^{43,44}$

Debido a que la prevalencia de ACV en mujeres jóvenes es baja, el riesgo en jóvenes migrañosas también sigue siendo baja (17/100.000 a 19/100.000 mujeres por año) en ausencia de otros factores de riesgo. Un estudio epidemiológico a gran escala de los Países
Bajos mostró que las pacientes con MG en especial aquellas con MG con aura, tenían un perfil de riesgo cardiovascular menos favorable en comparación con los controles. ${ }^{45}$

Como estos estudios clínicos han sido apoyados mediante neuroimágenes, se ha visto que los pacientes con MG tenían mayor prevalencia de infartos cerebelosos silenciosos en comparación con los controles de la misma edad y sexo. ${ }^{46}$ También se asoció con un aumento de lesiones profundas de la sustancia blanca en las mujeres pero no en hombres.

Los anticonceptivos orales con 50 mcg o más de estrógeno aumentan el riesgo de $\mathrm{ACV}$ isquémico en mujeres jóvenes más de doce veces en casos con aura. ${ }^{42,47} \mathrm{El}$ riesgo relativo promedio, en comparación con las no usuarias es de 4.1; con dosis bajas de anticonceptivos orales el riesgo es menor y en algunos estudios no hay diferencia significativa. ${ }^{48}$ Se sabe que estos medicamentos son un factor de riesgo para la trombosis venosa cerebral ${ }^{49}$; también hay un leve aumento del riesgo de hemorragia subaracnoidea. ${ }^{50} \mathrm{La}$ asociación de ACV con el uso de triptanes no se ha encontrado hasta el momento. ${ }^{51}$ En cualquier caso, estos deben usarse con precaución, tomando en cuenta los riesgos cardio y cerebrovasculares individuales.

La decisión de prescribir anticonceptivos orales a una migrañosa debe ser individualizada y la relación riesgo/beneficio considerada. La mayoría de las mujeres jóvenes con MG sin aura pueden tomar dosis bajas (35 mcg o menos de etinil estradiol diarios) combinados en forma segura a menos que tengan otros factores de riesgo vascular. Un aura de nueva aparición o un cambio dramático en una establecida obliga a retirar los anticonceptivos orales que contienen estrógenos ${ }^{42}$ y se deben ofrecer métodos no estrogénicos. Las pacientes deben ser advertidas de abstenerse o dejar de fumar.

La MG en sí no aumenta el riesgo de dar a luz a niños con deformidades ${ }^{52,53}$, no difieren en la prevalencia de abortos involuntarios, toxemia, anomalías congénitas o nacidos muertos. ${ }^{54}$ Sin embargo, en un estudio italiano de control de caso las embarazadas que tenían preeclampsia eran más propensas a reportar una historia de 
MG sin aura que las mujeres control con embarazos sin complicaciones ${ }^{55}$ La mejoría es más común en MG menstrual que en la no menstrual. En quienes la MG comenzó en la menarquia tienen una tasa de remisión más alta que cuando los dolores de cabeza aparecieron en otros momentos.

Después de la menopausia cuando los niveles de hormonas sexuales se estabilizan, la mayoría mejoran la MG y cuando es fisiológica experimentan una evolución más favorable que si la menopausia es quirúrgica. ${ }^{5}$ Si la MG empeora con la terapia de reemplazo hormonal convencional, los moduladores selectivos del receptor de estrógeno pueden ser apropiados con el raloxifeno que ha demostrado aumento de la densidad mineral ósea y reducción del colesterol total y las lipoproteínas de baja densidad, sin estimular el endometrio. ${ }^{56}$

\section{Tratamiento}

Migraña menstrual: la profilaxis solo es posible en pacientes con ciclos regulares y se inicia tres días antes de cada menstruación, durante la misma e incluso en algunos casos hasta tres días después. De los medicamentos más utilizados está el naproxeno en dosis de $500 \mathrm{mg}$ cada $12 / \mathrm{h}$ y se recomienda probar varios AINES hasta encontrar el más eficaz para cada paciente. Los derivados de la ergotamina son apropiados como profilaxis si se administran en los días anteriores a la menstruación, previa exclusión de los riesgos cardiovasculares. No hay estudios que demuestren la eficacia del tratamiento profiláctico con triptanes por vía oral cada ocho o doce horas en MG menstrual.

La profilaxis hormonal se utiliza cuando han fallado otras terapias y puede usarse cuando no hay contraindicaciones para el tratamiento con estrógenos como en MG con aura, diabetes, hipertensión arterial o tabaquismo. El efecto terapéutico se logra al mantener las concentraciones de estrógenos a lo largo del ciclo con la administración de ACO en dosis bajas de estrógenos (30 o $50 \mu \mathrm{g}$ ) asociado o no con progesterona. Al comienzo se toman durante tres semanas y se suspenden durante una para que se produzca la menstruación. Si persiste la MG menstrual durante la semana que se interrumpe la píldora, se pueden administrar los ACO en forma ininterrumpida durante tres o cuatro meses. ${ }^{42}$

\section{Embarazo}

La mayoría de los dolores de cabeza que ocurren durante el embarazo son cefaleas primarias en especial MG y tipo tensión. El uso de medicamentos puede aumentar el riesgo de abortos espontáneos, anormalidades del crecimiento fetal, complicaciones perinatales y teratogenicidad, efectos adversos que dependen del medicamento usado en relación con los periodos de desarrollo. La FDA (Food and Drugs Administration System) clasifica a los medicamentos usados en el embarazo en cinco categorías, con el fin de brindar una orientación terapéutica; A) estudios controlados en humanos no muestran ningún riesgo; B) sin evidencia de riesgo en los seres humanos, pero no hay estudios controlados en humanos; C) no se han descartado riesgos para los seres humanos; D) evidencia positiva de riesgo de estudios en humanos y/o animales; y $\mathbf{X}$ ) está contraindicado en el embarazo. ${ }^{57}$ Por otro lado existe otro sistema de categorización para evaluar la seguridad de los medicamentos durante el embarazo llamado TERIS (The Teratogen Information System) orientado a indicar riesgo alto, moderado, leve, mínimo y ningún riesgo..$^{58}$

En el tratamiento agudo de los ataques de MG es necesario hacer un abordaje individual, comenzando con descanso y medidas térmicas, evitando los desencadenantes. Ante los dolores de cabeza que no responden a las medidas no farmacológicas, están indicados los medicamentos sintomáticos como AINES y acetaminofén (solo o con codeína). Los opioides pueden producir adicción materna y neonatal, y su uso durante largos períodos y en dosis elevadas está contraindicado. La administración regular de analgésicos opiáceos o no tres o más días a la semana durante al menos seis semanas, genera que el dolor de cabeza empeore y se torne refractario al tratamiento. El uso crónico diario de opioides durante los últimos trimestres del embarazo se cuestiona debido a los riesgos de mortalidad fetal y parto prematuro. ${ }^{59}$ Dentro de los medicamentos disponibles para el manejo agudo del dolor se encuentra la aspirina que en dosis intermitentes bajas no tiene 
riesgo teratogénico significativo, aunque en dosis grandes se han asociado con hemorragias materno fetales.

Los antiinflamatorios no esteroideos AINES se pueden tomar para el dolor durante el primer trimestre del embarazo, pero la exposición temprana se ha asociado con abortos. ${ }^{60} \mathrm{Su}$ uso debe limitarse después de la semana 32, ya que algunos AINES pueden cerrar el ductus arterioso fetal. ${ }^{58,61,62}$ Para evitar lo anterior, como alternativa se encuentra el acetaminofén que no tiene los efectos adversos anteriores. En Estados Unidos se encuentra restringido el uso de AINES solo para el tercer trimestre del embarazo, aunque en algunos países europeos lo hacen desde el segundo. ${ }^{63}$

La cafeína puede ser una terapia adyuvante útil para aumentar el efecto analgésico del acetaminofén y está clasificada por la FDA en la categoría B. En un estudio la adición de $100 \mathrm{mg}$ de cafeína al analgésico incrementó en 1,5 veces el número de personas sin dolor en MG sin aura ${ }^{64}$ Se prefieren los productos que combinan acetaminofén y cafeína sólo durante el embarazo. Por otra parte, las pacientes pueden complementar el tratamiento analgésico con acetaminofén adicionando una bebida que contenga cafeína, como por ejemplo un café expresso de dos onzas que posee $100 \mathrm{mg}$ de cafeína. En una revisión reciente de la literatura no se identificó aumento del riesgo de malformación asociado con la exposición a cafeína materna. ${ }^{65}$

Otro gran estudio epidemiológico evaluó el consumo de cafeína materna en 4.196 bebés con malformaciones cardiovasculares y 3.957 niños sanos, controlando la exposición a nicotina, alcohol, medicamentos vasoactivos y suplementos de ácido fólico. No hay asociaciones positivas significativas identificadas entre el consumo de cafeína materna y el riesgo de malformación cardiovascular en los bebés expuestos. ${ }^{66}$ Los inhibidores más potentes de la síntesis de prostaglandinas, tales como salicilatos e indometacina, deben evitarse en lo posible durante el embarazo y desde luego en el último trimestre.

En cuanto a los barbitúricos y las benzodiazepinas su uso debe limitarse como también en mayor medida la ergotamina y la dihidroergotamina que se encuentran dentro de la categoría X y aunque tienen un efecto oxitócico débil es mejor evitar cualquier complicación natural del embarazo que podría estar atribuida a su uso, ya que se ha descrito parto pretérmino asociado. ${ }^{67,68}$ Todos los triptanes se categorizan por la FDA como clase $\mathrm{C}$ en el embarazo, que significa que "la seguridad en el embarazo humano no ha sido determinada" y que " los potenciales beneficios deben justificar los potenciales riesgos". Los triptanes es mejor evitarlos, aunque esta creencia ha sido controvertida. ${ }^{68}$

Los corticosteroides se pueden utilizar de vez en cuando (algunos sugieren limitar su uso durante el primer trimestre). Es una manera eficaz de manejar el estado migrañoso durante el embarazo, así como el uso de sulfato de magnesio intravenoso (categoría B) que en dosis de dos gramos ha demostrado ser efectivo en el manejo de dolor agudo. ${ }^{1,6,10}$ Otra opción es la lidocaína en solución nasal al 4\% (categoría B) aplicando 0.5 a $1 \mathrm{ml}$ por la fosa nasal del lado del dolor de cabeza. Se puede repetir dos minutos después si es necesario. ${ }^{69,70}$

Para el manejo sintomático de las náuseas se puede usar solución de carbohidratos fosforilados (emetrol, una solución oral que contiene cantidades equilibradas de fructosa y glucosa con ácido ortofosfórico) o succinato de doxilamina y vitamina B6 (piridoxina). Las náuseas persistentes pueden requerir inyecciones o supositorios tales como clorpromazina, proclorperazina o prometazina, disponibles por vía oral, parenteral y en forma de supositorio. Las dos últimas se utilizan con frecuencia en países europeos en supositorios.

En agudos ataques severos de MG se debe iniciar el manejo en forma agresiva. Como primera medida garantizar una adecuada hidratación con líquidos y luego administrar metoclopramida $10 \mathrm{mg}$ IV para controlar tanto las náuseas y el dolor de cabeza, o bien ondansetron siendo el de elección por mayor seguridad y mejor tolerancia (categoría B) en el embarazo en dosis de 4 a 8 mg IV en 2 o 3 minutos. ${ }^{58}$ Los opioides intravenosos como hidromorfona 0-5-1 mg en 2 a 3 minutos y tramadol $100 \mathrm{mg}$ IM (categoría C) también son útiles. 
Dentro de la terapia de rescate juegan un papel importante los opiodes, ya que los triptanes y la dihidroergotamina están restringidos en embarazadas; estos deben usarse en forma cautelosa e individualizando cada paciente. ${ }^{71}$ La realización de bloqueos con lidocaína y dexametasona en puntos gatillo y bloqueos del nervio occipital, pueden ser eficaces durante el embarazo. Como norma general, durante la preñez deben evitarse los tratamientos profilácticos. En los casos estrictamente necesarios, los betabloqueadores son los fármacos más seguros, en especial el propanolol y el labetalol, suspendiendo su administración dos semanas antes del parto. El verapamilo parece ser un fármaco seguro durante el segundo y el tercer trimestre.

Es importante considerar la opción de manejo preventivo cuando se presentan tres o cuatro ataques al mes que son incapacitantes o sin respuesta a la terapia sintomática, ya que puede ocasionar deshidratación y agresión fetal. Los betabloqueadores como el propanolol se pueden usar, pero tiene efectos adversos como retardo en el crecimiento intrauterino y oligohidramnios. ${ }^{72}$ Dentro de los medicamentos para el manejo preventivo de la MG en el embarazo, teniendo en cuenta el riesgo y las comorbilidades que requieran estricto manejo farmacológico, se encuentran los antidepresivos tricíclicos como amitriptilina (categoría C), otros del tipo de inhibidores de la recaptación de serotonina como citalopram, duloxetina, fluoxetina, venlafaxina (categoría C) exceptuando la sertralina (categoría B) y por último los antiepilépticos gabapentin y topiramato (categoría C) excepto el ácido valproico (categoría D, evidencia positiva de riesgo en humanos). Los anticonvulsivantes son teratogénicos potenciales y tienen efectos leves en las funciones cognitivas de las gestantes y grados variables de sedación. ${ }^{73}$

Los suplementos naturales como el magnesio (100 a 400 mg día) y la vitamina B2 han demostrado beneficio en los estudios doble ciego para la profilaxis de la MG y se pueden utilizar cuando sea necesario. La riboflavina $400 \mathrm{mg} /$ día es eficaz en el manejo preventivo de MG con aura pero tiene un inicio de acción lento y su efecto máximo sobre la frecuencia de los ataques se produce después de tres meses de tratamiento. Se considera el fármaco de primera elección en pacientes con una frecuencia por debajo de cuatro a cinco ataques al mes y no en forma severa. Se desconocen los efectos teratogénicos de la riboflavina. ${ }^{74}$

Otras opciones no farmacológicos incluyen biofeedback, (técnicas de retroalimentación) terapia física, acupuntura, yoga, higiene del sueño y evitar los desencadenantes identificados por cada paciente como estrés, bebidas, ayuno, alcohol, olores fuertes, etc..$^{57,75}$ Las migrañas cesan en el segundo y tercer trimestre del embarazo, en dos tercios de las mujeres. Si se demuestra que los agentes utilizados en el tratamiento son perjudiciales para el feto, se debe suspender la medicación tan pronto como sea posible. ${ }^{67}$

\section{Menopausia}

El reemplazo hormonal es el tratamiento de elección en las mujeres migrañosas perimenopáusicas y su objetivo es mantener niveles bajos sostenidos de estrógeno. Muchas reportan grados variables de mejoría clínica, mientras otras no ven modificación en la misma o sufren un empeoramiento en especial si la MG es con aura.

Los estrógenos se pueden administrar por vía oral, transdérmica o vaginal y deben asociarse con progestágenos para reducir el riesgo de cáncer de endometrio. Siempre debe utilizarse la dosis mínima eficaz. La clonidina (75 mcg/día) es una interesante opción terapéutica en mujeres menopáusicas migrañosas y previene algunos síntomas atribuidos al descenso de estrógenos. Esta revisión referencia medicamentos y medidas terapéuticas que se disponen durante la menstruación, embarazo y menopausia, su elección debe ser individualizada para cada etapa y decidida en conjunto con la paciente.

\section{Referencias}

1. Goadsby PJ, Lipton RB, Ferrari MD. Migraine--current understanding and treatment. N Engl J Med. 2002; 346(4):257-70.

2. Nagel-Leiby S, Welch KM, Grunfeld S, D'Andrea G. Ovarian steroid levels in migraine with and without aura. Cephalalgia 1990; 10(3):147-52. 
3. Somerville BW. The role of progesterone in menstrual migraine. Neurology. 1971; 21(8):853-9.

4. MacGregor EA, Frith A, Ellis J, Aspinall L, Hackshaw A. Incidence of migraine relative to menstrual cycle phases of rising and falling estrogen. Neurology. 2006; 67(12):2154-8.

5. MacGregor EA. M Menstruation, sex hormones, and migraine. Neurol Clin. 1997; 15(1):125-41.

6. Somerville BW. Estrogen-withdrawal migraine. II. Attempted prophylaxis by continuous estradiol administration. Neurology. 1975; 25(3):245-50.

7. Somerville BW. Estrogen-withdrawal migraine. I. Duration of exposure required and attempted prophylaxis by premenstrual estrogen administration. Neurology. $1975 ; 25(3): 239-44$

8. Somerville BW. The role of estradiol withdrawal in the etiology of menstrua migraine. Neurology. 1972; 22(4):355-65.

9. Puri V, Puri S, Svojanovsky SR, Mathur S, Macgregor RR, Klein RM, et al. Effects of oestrogen on trigeminal ganglia in culture: implications for hormonal effects on migraine. Cephalalgia. 2006; 26(1):33-42.

10. Martin V, Behbehani M. Fos expression of trigeminal nucleus caudalis neurons after dural activation during different states of the rat estrous cycle [abstract] Headache. 2005; 45:788.

11. Gangula PR, Zhao H, Wimalawansa SJ, Supowit SC, DiPette DJ, Yallampalli C. Pregnancy and steroid hormones enhance the systemic and regional hemodynamic effects of calcitonin gene-related peptide in rats. Biol Reprod. 2001; 64(6):1776-83

12. Kim GM, Jin KS, Chung CS. Differential effects of corticosteroids on the expression of cyclooxygenase-2, tumour necrosis factor-alpha and matrix metalloproteinase-9 in an animal model of migraine. Cephalalgia. 2008; 28(11):1179-87.

13. Sicuteri F. Opioids, pregnancy and the disappearance of headache. Headache. 1980; 20(4):220-1.

14. Sakai Y, Dobson C, Diksic M, Aube M, Hamel E. Sumatriptan normalizes the migraine attack-related increase in brain serotonin synthesis. Neurology. 2008 70(6):431-9.

15. Bethea CL, Lu NZ, Gundlah C, Streicher JM. Diverse actions of ovarian steroids in the serotonin neural system. Front Neuroendocrinol. 2002; 23(1):41-100.

16. Finocchi C, Ferrari M. Female reproductive steroids and neuronal excitability. Neurol Sci. 2011; 32 Suppl 1:S31-5.

17. Theoharis TC, Donelan J, Kandere-Grzybowska K, Konstantinidou A. The role of mast cells in migraine pathophysiology. Brain Res Brain Res Rev. 2005; 49(1):65-76.

18. Boes T, Levy D. Influence of sex, estrous cycle and estrogen on intracranial dura mast cells. Cephalalgia 2012; 32(12):924-31.

19. Magos A, Brincat M, Zilkha KJ, Studd JW. Serum dopamine beta-hydroxylase activity in menstrual migraine. J Neurol Neurosurg Psychiatry. 1985; 48(4):328 31 .

20. Facchinetti F, Martignoni E, Fioroni L, Sances G, Genazzani AR. Opioid control of the hypothalamus- pituitary adrenal axis cyclically fails in menstrual migraine. Cephalalgia 1990; 10(1):51-6.

21. Facchinetti F, Sances G, Borella P, Genazzani AR, Nappi G. Magnesium prophylaxis of menstrual migraine: effects on intracellular magnesium. Headache. 1991; 31(5):298-301.

22. Headache Classification Committee of the International Headache Society (IHS). The International Classification of Headache Disorders. 3rd ed. (beta version). Cephalalgia. 2013; 33(9):629-808

23. Granella F, Sances G, Allais G, Nappi RE, Tirelli A, Benedetto C, et al. Characteristics of menstrual and nonmenstrual attacks in women with menstrually related migraine referred to headache centres. Cephalalgia. 2004; 24(9):707-16.

24. Boyle CA. Management of menstrual migraine. Neurology 1999; 53(4 Suppl 1):S14-8.

25. Keenan PA, Lindamer LA. Non-migraine headache across the menstrual cycle in women with and without premenstrual syndrome. Cephalalgia. 1992; 12(6):3569; discussion 339
26. MacGregor EA, Chia H, Vohrah RC, Wilkinson M. Migraine and menstruation: a pilot study. Cephalalgia 1990; 10(6):305-10.

27. MacGregor EA, Igarashi H, Wilkinson M. Headaches and hormones: subjective versus objective assessment. Headache Q 1997 b;8:126-36.

28. Maggioni F, Alessi C, Maggino T, Zanchin G. Headache during pregnancy. Cephalalgia. 1997; 17(7):765-9.

29. Sances G, Granella F, Nappi RE, Fignon A, Ghiotto N, Polatti F et al. Course of migraine during pregnancy and postpartum prospective study. Cephalalgia. 2003; 23(3):197-205

30. Bousser MG. Estrogens, migraine, and stroke. Stroke. 2004; 35(11 Suppl 1):2652-6

31. Melhado E, Maciel JA Jr, Guerreiro CA. Headaches during pregnancy in women with a prior history of menstrual headaches. Arq Neuropsiquiatr. 2005; 63(4):934-40.

32. MacGregor EA. Menstruation, sex hormones, and migraine. Neurol Clin. 1997; 15(1):125-41.

33. Wall VR. Breastfeeding and migraine headaches. J Hum Lact. 1992; 8(4):209-12.

34. Meriggiola MC, Nanni M, Bachiocco V, Vodo S, Aloisi AM. Menopause affects pain depending on pain type and characteristics. Menopause 2012; 19(5):517-23.

35. Fettes I. Migraine in the menopause. Neurology. 1999; 53(4 Suppl 1):S29-33.

36. Neri I, Granella F, Nappi R, Manzoni GC, Facchinetti F, Genazzani AR. Characteristics of headache at menopause: a clinico-epidemiologic study. Maturitas. 1993; 17(1):31-7.

37. Mueller L. Predictability of exogenous hormone effect on subgroups of migraineurs. Headache. 2000; 40(3):189-93.

38. Greendale GA, Reboussin BA, Hogan P, et al. Symptom relief and side effects of postmenopausal hormones: results from the postmenopausal Estrogen/Progestin Interventions Trial. Obstet Gynecol. 1998; 92(6):982-8.

39. Misakian AL, Langer RD, Bensenor IM, Cook NR, Manson JE, Buring JE, Rexrode KM. Postmenopausal hormone therapy and migraine headache. J Womens Health (Larchmt). 2003; 12(10):1027-36.

40. Martin VT, Behbehani M. Ovarian hormones and migraine headache: understanding mechanisms and pathogenesis, part I. Headache. 2006; 46(1):3-23.

41. Martin VT, Behbehani M. Ovarian hormones and migraine headache: understanding mechanisms and pathogenesis, part 2. Headache. 2006; 46(3):365-86.

42. Becker WJ. Use of oral contraceptives in patients with migraine. Neurology 1999; 53(4 Suppl 1):S19-25.

43. Kurth T1, Slomke MA, Kase CS, Cook NR, Lee IM, Gaziano JM, et al. Migraine, headache, and the risk of stroke in women: a prospective study. Neurology. 2005; 64(6):1020-6.

44. Katsarava Z, Rabe K, Diener HC. From migraine to stroke. Intern Emerg Med. 2008; 3 Suppl 1:S9-16.

45. Scher AI, Terwindt GM, Picavet HS, Verschuren WM, Ferrari, MD, Launer LJ. Cardiovascular risk factors and migraine: the GEM population-based study. Neurology. 2005; 64(4):614-20.

46. Kruit MC, van Buchem MA, Hofman PA, Bakkers JT, Terwindt GM, Ferrari MD, et al. Migraine as a risk factor for subclinical brain lesions. JAMA. 2004; 291(4):427-34

47. World Health Organization. Ischemic stroke and combined oral contraceptives: results of an international, multicenter, case-control study. Lancet. 1996; 348(9026):498-505.

48. Siritho S, Thrift AG, McNeil JJ, You RX, Davis SM, Donnan GA, et al. Risk of ischemic stroke among users of the oral contraceptive pill: The Melbourne Risk Factor Study (MERFS) Group. Stroke. 2003; 34(7):1575-80.

49. Bousser MG, Kittner SJ. Oral contraceptives and stroke. Cephalalgia. 2000; 20(3):183-9.

50. Johnston SC, Colford JM, Gress DR. Oral contraceptives and the risk of subarachnoid hemorrhage: a meta-analysis. Neurology. 1998; 51(2):411-8. 
51. Hall GC, Brown MM, Mo J, MacRae KD. Triptans in migraine: the risks of stroke, cardiovascular disease, and death in practice. Neurology. 2004; 62(4):563-8.

52. Wainscott G, Sullivan FM, Volans GN, Wilkinson M. The outcome of pregnancy in women suffering from migraine. Postgrad Med J. 1978; 54(628): 98-102.

53. Aube M. Migraine in pregnancy. Neurology. 1999; 53(4 Suppl 1):S26-8.

54. Silberstein SD. Headaches in pregnancy. Neurol Clin. 2004; 22(4):727-56.

55. Facchinetti F, Allais G, D'Amico R, Benedetto C, Volpe A. The relationship between headache and preeclampsia: a case-control study. Eur J Obstet Gynecol Reprod Biol. 2005; 121(2):143-8.

56. Delmas PD, Bjarnason NH, Mitlak BH, Ravoux AC, Shah AS, Huster WJ, et al. Effects of raloxifene on bone mineral density, serum cholesterol concentrations, and uterine endometrium in postmenopausal women. N Engl J Med. 1997; 337(23):1641-7.

57 Young WB, Silberstein SD, Nahas SJ, Marmura MJ. Jefferson Headache Manual New York: Demos Medical; 2011.

58. Marcus DA, Bain PA. Treatment of pregnant and breastfeeding patients with acute headache in the ED. Practical assessment and treatment of the patient with headaches in the Emergency Department and Urgent Care Clinic: New York: Springer; 2011. p. 133-54.

59. Beers MH, Berkow R. The Merck Manual of Diagnosis and Therapy. 18th ed. Whitehouse Station: Merck Research Laboratories, 2006.

60. Li DK, Liu L, Odouli R. Exposure to non-steroidal anti-inflammatory drugs during pregnancy and risk of miscarriage: population based cohort study. BMJ. 2003; 327(7411):368.

61. Levin M, Comprehensive review of headache medicine. New York: Oxford University Press; 2008.

62. Cete Y, Dora B, Ertan C, Ozdemir C, Oktay C. A randomized prospective placebo-controlled study of intravenous magnesium sulphate vs. metoclopramide in the management of acute migraine attacks in the Emergency Department. Cephalalgia. 2005; 25(3):199-204.

63. Evers S, Afra J, Frese A, Goadsby PJ, Linde M, May A, et al. EFNS guideline on the drug treatment of migraine--revised report of an EFNS task force. Eur J Neurol. 2009; 16(9):968-81.
64. Peroutka SJ, Lyon JA, Swarbrick J, Liption RB, Kolodner K, Goldstein J. Efficacy of diclofenac sodium softgel $100 \mathrm{mg}$ with or without caffeine $100 \mathrm{mg}$ in migraine without aura: a randomized, double-blind, crossover study. Headache. 2004; 44(2):136-41.

65. Browne ML. Maternal exposure to caffeine and risk of congenital anomalies: a systematic review. Epidemiology. 2006; 17(3):324-31.

66. Browne ML, Bell EM, Druschel CM, Gensburg LJ, Mitchell AA, Lin AE, et al. Maternal caffeine consumption and risk of cardiovascular malformations. Birth Defects Res A Clin Mol Teratol. 2007; 79(7):533-43.

67. Lance JW, Goadsby PJ. Mechanism and Management of Headache. 7th ed. Philadelphia : Elsevier; 2005.

68. Loder E. Safety of sumatriptan in pregnancy: a review of the data so far. CNS Drugs. 2003; 17(1):1-7.

69. Maizels M, Scott B, Cohen W, Chen W. Intranasal lidocaine for treatment of migraine: a randomized, double-blind, controlled trial. JAMA. 1996; 276(4):31921.

70. Blanda M, Rench T, Gerson LW, Weigand JV. Intranasal lidocaine for the treatment of migraine headache: a randomized, controlled trial. Acad Emerg Med. 2001; 8(4):337-42

71. Tornabene SV, Deutsch R, Davis DP, Chan TC, Vilke GM. Evaluating the use and timing of opioids for the treatment of migraine headaches in the emergency department. J Emerg Med. 2009; 36(4):333-7.

72. Lane R, Davies P. Migraine. New York: Taylor \& Francis Group; 2006.

73. Lipton RB, Bigal ME. Migraine and other headache disorders. New York: Informa Healthcare; 2006

74. Olesen J, Silberstein SD, Tfelt-Hansen P. Preventive pharmacotherapy of headache disorders. New York: Oxford University Press; 2004.

75. Schulman EA, Levin M. Refractory Migraine: Mechanisms and Management New York: Oxford University Press; 2010 